\title{
Eliciting and measuring betrayal aversion using the BDM mechanism
}

\author{
Simone Quercia ${ }^{1}$
}

Received: 10 April 2015/Revised: 16 December 2015/Accepted: 21 December 2015/

Published online: 11 January 2016

(C) Economic Science Association 2016

\begin{abstract}
Betrayal aversion has been operationalized as the evidence that subjects demand a higher risk premium to take social risks compared to natural risks. This evidence has been first shown by Bohnet and Zeckhauser ( $\mathrm{J}$ Econ Behav 98:294-310, 2004) using an adaptation of the Becker-DeGroot-Marschak mechanism (BDM, Becker et al. Behav Sci 9:226-232, 1964). We compare their implementation of the BDM mechanism with a new version designed to facilitate subjects' comprehension. We find that, although the two versions produce different distributions of values, the size of betrayal aversion, measured as an average treatment difference between social and natural risk settings, is not different across the two versions. We further show that our implementation is preferable to use in practice as it reduces substantially subjects' mistakes and the likelihood of noisy valuations.
\end{abstract}

Keywords Experiments - Betrayal aversion - Trust game - Becker-DeGrootMarschak mechanism · Preference elicitation

JEL Classification C90 - D81

Electronic supplementary material The online version of this article (doi:10.1007/s40881-015-0021-3) contains supplementary material, which is available to authorized users.

Simone Quercia

simone.quercia@uni-bonn.de

1 Institute for Applied Microeconomics (IAME), University of Bonn, Adenauerallee 24-42, 53113 Bonn, Germany 


\section{Introduction}

Recent experimental evidence shows that people demand a higher risk premium when the source of risk is another person rather than nature (see Bohnet and Zeckhauser 2004; Bohnet et al. 2008). Moreover, they prefer to opt out from a trust game and let their earnings be determined by a payoff-equivalent lottery to avoid learning whether their co-player betrayed them (Aimone and Houser 2011, 2012, 2013). This evidence reveals that people anticipate the psychological cost of betrayal that they suffer on top of monetary costs.

This phenomenon, commonly referred to as betrayal aversion, has been first investigated by Bohnet and Zeckhauser (2004) (BZ, henceforth) using an adaptation of the Becker-DeGroot-Marschak mechanism (BDM, see Becker et al. 1964). Their design compares so-called minimum acceptance probabilities (MAPs) between a social risk and a natural risk situation. A MAP is defined as the minimum probability of the good outcome that subjects require to choose a risky bet rather than a certain payoff. The finding that MAPs are on average higher in the social risk compared to the natural risk setting has been interpreted as evidence that subjects are betrayal averse.

In this paper, we ask whether different implementations of the BDM mechanism affect the elicitation of values and the measurement betrayal aversion in the BZ design. Our question is particularly important for two main reasons. First, recent evidence shows that the BDM mechanism may be empirically not reliable due to its complexity and to subjects' misconceptions of the incentive structure (see, e.g., Cason and Plott 2014). Second, the MAP design uses the BDM mechanism in an unusual way compared to the previous literature as it elicits a probability instead of a price. In most applications, the BDM mechanism is used in selling or buying tasks and subjects are asked the minimum price demanded to give up an object or the maximum price they would be willing to pay to buy the object. The MAP design, instead, asks subjects to reveal the minimum value of a probability that they would require to play a lottery. Arguably this may further increase the complexity of the mechanism. ${ }^{1}$

In our experiment we simplify the original BZ instructions and procedures to minimize the risk of misconceptions and ask whether this has an impact on the elicitation of betrayal aversion. Study 1, reported in Sect. 2, consists of two experimental conditions: the open-ended (OE) condition replicates the design and procedures of $\mathrm{BZ}$, whereas the choice list (CL) condition introduces a novel instrument and procedures intended to facilitate subjects' comprehension.

Our results from Study 1 show that the way the BDM mechanism is presented to subjects has a significant impact on elicited values. The two elicitation methods generate statistically different distributions of data, with the CL distribution of MAPs being more peaked and presenting less variation. However, when we compare the estimated size of betrayal aversion (the average difference of MAPs in

\footnotetext{
1 Previous experimental literature has long recognized the possibility of subjects' confusion and mistakes (see Andreoni 1995; Houser and Kurzban 2002 for examples in public goods games). In the specific context of the BDM mechanism, recent contributions are Hao and Houser (2012), Cason and Plott (2014) and Bartling et al. (2015).
} 
the social versus the natural risk situation), we find no differences between $\mathrm{OE}$ and CL.

We ask next which method is preferable to use in terms of truthful revelation. To answer this question, in Study 2, we conduct a survey with a sample of undergraduate students, where we compare the experimental instructions of $\mathrm{OE}$ and CL used in Study 1 on the basis of perceived complexity and number of mistakes made in a set of comprehension questions. We show that our CL instructions are better understood than the $\mathrm{OE}$ instructions in terms of both perceived complexity and number of mistakes in the comprehension questions. We discuss the implication of these findings in our concluding section.

\section{Study 1: two versions of the BDM mechanism for the elicitation of betrayal aversion}

\subsection{Experimental design and procedures}

Our design consists of two treatments: the OE and the CL treatment. The OE treatment is an exact replication of $\mathrm{BZ} .^{2}$ The $\mathrm{CL}$ treatment introduces a new instrument, procedures and instructions to measure betrayal aversion.

Following BZ, each of our treatments permits a between-subjects comparison of two conditions called trust game (TG) and risky dictator game (RDG). ${ }^{3}$ Figure 1 depicts the extensive form of TG and RDG. In both decision tasks, a first mover chooses between a certain and a risky option. The certain option gives 10 points to them and 10 to their counterpart. The risky option can produce either an unequal outcome of 8 points to the first mover and 22 to their counterpart or an equal split giving 15 points to both. While in TG, the outcome of the risky option is determined by a second mover, in RDG, it is determined by nature with the probabilities of outcomes $(15 ; 15)$ and $(8 ; 22)$ being, respectively, $p$ and $1-p$. This is the only difference between the two conditions.

The variable of interest is the probability of the outcome $(15 ; 15)$ which makes first movers indifferent between the certain and the risky option. Following BZ, we shall call this value the minimum acceptance probability (MAP). If the average difference in elicited MAPs between $T G$ and RDG is positive, that is $\left(\overline{\mathrm{MAP}}_{\mathrm{TG}}-\overline{\mathrm{MAP}}_{\mathrm{RDG}}>0\right)$, subjects are on average betrayal averse as they require a higher risk premium in the social compared to the natural risk setting. In what follows, we describe our two treatments in detail.

\footnotetext{
${ }^{2}$ We use the instructions from the web appendix of Bohnet et al. (2008), a cross-cultural study on betrayal aversion that uses same instructions and procedures of BZ.

3 The original design is composed of three treatments called trust game, risky dictator game and decision problem. The comparison between the first and the second treatment measures betrayal aversion, while the comparison between the second and the third measures social preferences. As we are mainly interested in betrayal aversion, we implemented only the first two.
} 


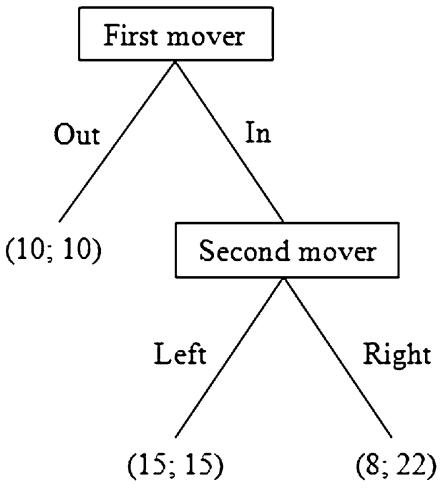

TG

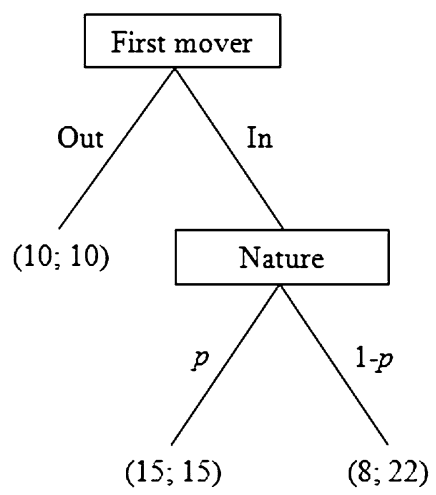

RDG

Fig. 1 Monetary payoffs in TG and RDG

\subsubsection{Open-ended (OE) elicitation of betrayal aversion (Bohnet and Zeckhauser 2004)}

Both in TG and RDG, each first mover is asked to state directly the MAP by writing it down on their answer sheet. In particular, in TG each first mover is asked the following question:

"Key question: how large would the probability $p$ of being paired with a person $\mathrm{Y}$ who chose option 1 minimally have to be for you to pick alternative $\mathrm{B}$ over alternative A? (like any probability it must lie between 0 and 1)." 4

At the same time, second movers are asked to reveal their strategy using the strategy method (Selten 1967), namely they are asked whether they would choose to be trustworthy or not in case their first mover chooses in (alternative B according to BZ labels). After collecting the answers, the experimenter computes the actual fraction of trustworthy second movers in the session and determines the decision of first movers according to their stated MAP. If their MAP is greater than the actual fraction of trustworthy second movers, they are deemed to choose Out (alternative A). If, instead, their MAP is less or equal than the actual fraction of trustworthy second movers, they are deemed to choose In (alternative B) and the outcome depends on their second mover's choice. This mechanism to determine first movers' action is known to first movers, but unknown to second movers. Second movers only know that first movers will make a choice between in and out.

In RDG, recipients are simply asked to wait for the decision of first movers. Each first mover is instead asked the following:

"Key question: how large would the probability $\mathrm{p}$ of the lottery producing option 1 minimally have to be for you to pick alternative B over alternative A? (like any probability, it must lie between 0 and 1)."

\footnotetext{
${ }^{4}$ In the OE treatment, we used the original labeling of strategies of Bohnet et al. (2008) (see Online Appendix A.2 for the full set of instructions). For the ease of exposition, in the remainder of the paper we always use the labels of Fig. 1, which correspond to our CL treatment.
} 
The mechanism to determine outcomes is similar to TG except that the elicited MAP is compared with a probability $p$ which is predetermined before the experiment and unknown to subjects. If the stated MAP is greater than $p$, the subject is deemed to choose out (alternative A), while if the MAP is less or equal than $p$, the subject is deemed to choose In (alternative B). At the end of the session the experimenter reveals the actual value of $p$ and the lottery is implemented.

The advantage of the open-ended version is that it is fast to implement and it elicits values with high precision, because subjects can state "point" valuations. The main disadvantage is that, in standard implementations like selling or buying tasks, it is not clear whether subjects fully understand the functioning of the bidding mechanism (see Harrison and Rutstrom 2008). In the context of probability elicitation, there is the risk that the mechanism and the wording of the "key question" may be even more obscure to subjects compared to standard implementations. For example, in a selling task the typical question would be something like "What is the lowest price at which you would sell?" which less likely creates comprehension problems compared to the probability elicitation setting described above.

\subsubsection{Our innovation: choice list (CL) elicitation of betrayal aversion}

The procedure used to elicit the decisions of second movers is identical to the $\mathrm{OE}$ treatment. First movers, instead, are presented with a choice list table. In TG, the first column of the table reports all the possible fraction of second movers choosing Left from " 20 out of 20 " to " 0 out of 20 " for a total of 21 rows. In the second column, subjects are asked to circle In or Out for each row. In RDG, everyone is told that a draw from an urn with a predetermined number of green and yellow balls will determine the outcome for the subjects who choose In. The first column of the table displays all the possible fractions of yellow balls (see Online Appendix A.3 for an example of the table). First movers have to circle In or Out for each possible number of yellow balls in the urn. It is further explained to subjects that only one row is relevant for earnings (the one corresponding to the actual number of trustworthy second movers or to the actual number of yellow balls), but that they will know which row is relevant only after they have taken their decisions.

We expect subjects to choose In in the first row of the table when the outcome $(15 ; 15)$ is certain and switch to Out when the risk of being the sucker [unlucky] increases. We infer the MAPs from subjects' choices by taking the middle point between the last probability for which they choose In and the first probability for which they choose Out going from the top to the bottom of the table. ${ }^{5}$

\footnotetext{
5 We decided not to force subject to switch only once in the table. In the data analysis, we will exclude subjects who have more than one switch as a default criterion, but we will report robustness checks showing that our results are robust to the inclusion of these subjects. We have 23 subjects (14.3\% of our sample) reporting more than one switch. Among these subjects, we are not able to infer a MAP for two subjects $(1.2 \%$ of our sample) who violate dominance starting from Out in the first row of the table. For the remaining 21 subjects we can infer a MAP by taking the average between the first row that the subject switched at and the last row that the subject switched at. This approach assumes that multiple switching is the result of indifference between the choice list options in the interval where multiple switching occurs (see Andersen et al. 2006).
} 
The CL treatment varies three elements compared to the OE treatment: the choice list format, the descriptions of second movers' actions as frequencies instead of probabilities and the text of the instructions adding more detailed explanations and examples to make the mechanism as clear as possible (see Online Appendix A.2). The advantage of using the choice list format is that it is generally easy to explain to participants and it minimizes subjects' confusion. However, it has two potential disadvantages. First, it elicits only interval values rather than "point" valuations and is, thus, less precise than the open-ended method. Second, it can be prone to framing effects, such as for example, subjects' tendency to switch in the middle of the table rather than at their "true" value (see, e.g., Andersen et al. 2006). Regarding the presentation of probabilities as frequencies, evidence from psychology has shown that statistical reasoning is improved if problems are presented using frequencies rather than percentages or probabilities (see Gigerenzer and Hoffrage 1995). Thus, expressing the problem using frequencies may help subjects understand the functioning of the BDM mechanism. ${ }^{6}$

\subsubsection{Procedures}

All the sessions were conducted at the University of Nottingham. We used the instructions and control questions from Bohnet et al. (2008) for the OE treatment. ${ }^{7}$ We developed novel instructions and control questions for the CL treatment (see Online Appendix A.2). All the experimental sessions in both treatments were composed of several parts. In part 1, we conducted the experiment reported in this paper and in the other parts we conducted other experiments reported in a companion paper (Cubitt et al. 2015). Subjects knew of the existence, but no details about subsequent parts while they were completing part 1 .

Our sample is composed by undergraduate student participants from various disciplines recruited via ORSEE (Greiner 2015). Our sample was balanced across treatments in terms of relevant characteristics: in OE (CL) we had $50 \%(51 \%)$ females; $81 \%$ (82 \%) whose country of origin was a European country; average age was 20.4 (20.2); they participated on average in 3.5 (3.7) experiments before taking part in our sessions $(t$ test, $p$ value $=0.491)$. We conducted 16 sessions with 272 subjects in OE and 8 sessions with 320 subjects in CL. The OE and CL sessions were conducted 1 year apart (2011 and 2012, respectively) in the exact same period of the year. All outcomes were described as point earnings and converted at an exchange rate of $£ 0.2$ per point. Average hourly earnings were around $£ 8$.

\footnotetext{
${ }^{6}$ Our innovation changes the implementation of the BDM mechanism to improve subjects' comprehension. It does not address potential confounds that the original BZ method might have regarding the interpretation of the treatment difference between TG and RDG (we thank a referee for pointing this out). We refer the reader to BZ and Aimone and Houser (2012) for a discussion of these alternative interpretations. Aimone and Houser (2012) and Aimone et al. (2015) develop methods that solve these confounds.

7 We use the instructions from the web appendix of Bohnet et al. (2008) (http://www.aeaweb.org/aer/ data/mar08/20051024_app.pdf). We thank the authors for also making their control questions available.
} 

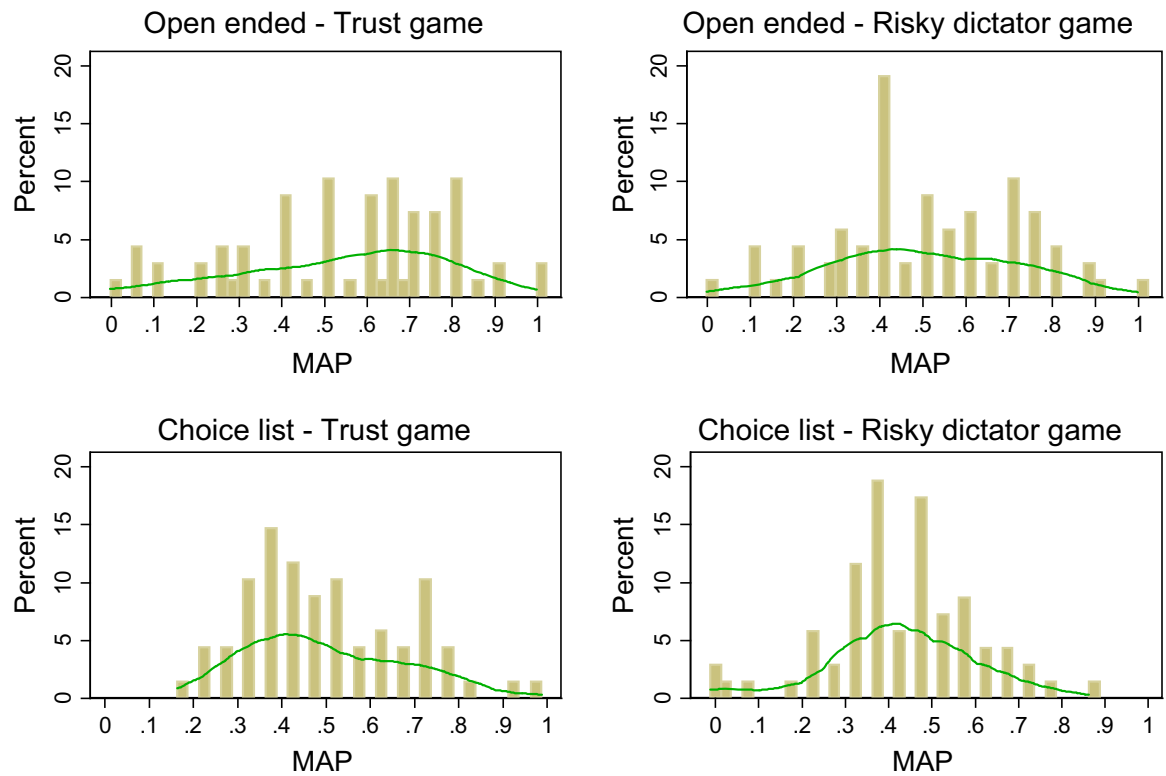

Fig. 2 Distribution of MAPs across treatments

\subsection{Experimental results}

Result 1 The two elicitation methods yield significantly different distributions of MAPs. CL generates significantly less variation and a more peaked distribution compared to OE.

Support The support for Result 1 comes from Fig. 2, where we show the histograms and estimated kernel density functions of MAPs in OE (two upper panels) and CL (two lower panels). The distribution of MAPs is statistically different across elicitation methods both in TG and RDG according to KolmogorovSmirnov tests ( $p=0.029$ and $p=0.042$, respectively). OE generates a less peaked distribution as suggested by the kernel density estimates and less dispersion around the mean as revealed by the significantly higher standard deviation in OE compared to CL (standard deviations are 0.23 and 0.18 , respectively; two-sided Levene test, $p=0.003$ ). This is true also looking separately at TG and RDG (standard deviations are 0.25 and 0.22 in $\mathrm{OE}$ and 0.18 and 0.17 in CL, respectively; two-sided Levene-tests, $p=0.012$ and $p=0.055$, respectively). ${ }^{8}$

Result 2 Betrayal aversion $\left(\overline{\mathrm{MAP}}_{\mathrm{TG}}-\overline{\mathrm{MAP}}_{\mathrm{RDG}}\right)$ is 0.04 in $\mathrm{OE}$ and 0.07 in CL. Econometric analysis reveals that the size of betrayal aversion is not significantly different between the two elicitation methods.

\footnotetext{
${ }^{8}$ Including the subjects with multiple switches in CL does not change our results both for the Kolmogorov-Smirnov tests ( $p=0.023$ and $p=0.016$ for TG and RDG, respectively) and for the Levene-tests ( $p=0.004$ and $p=0.021$ for TG and RDG, respectively).
} 
Table 1 Betrayal aversion in CL and OE treatments [mean (Std. dev.)]

\begin{tabular}{llll}
\hline & $\overline{\mathrm{MAP}}_{\mathrm{TG}}$ & $\overline{\mathrm{MAP}}_{\mathrm{RDG}}$ & $\overline{\mathrm{MAP}}_{\mathrm{TG}}-\overline{\mathrm{MAP}}_{\mathrm{RDG}}$ \\
\hline $\mathrm{CL}(n=137)$ & $0.50(0.18)$ & $0.43(0.17)$ & $0.07(0.17)^{*}$ \\
$\mathrm{OE}(n=136)$ & $0.54(0.25)$ & $0.50(0.22)$ & $0.04(0.23)$ \\
All $(n=273)$ & $0.52(0.22)$ & $0.47(0.20)$ & $0.05(0.21)^{* *}$ \\
\hline
\end{tabular}

** $p<0.05, * p<0.10$ according to two-sided Mann-Whitney $U$-test. Every MAP is treated as an independent observation. For the difference between MAPs, we report the pooled standard deviation

Support As a first step, in Table 1 we report the average MAP in each treatment for both methods separately and for the pooled sample. The size of betrayal aversion $\left(\overline{\mathrm{MAP}}_{\mathrm{TG}}-\overline{\mathrm{MAP}}_{\mathrm{RDG}}\right)$ is similar across the two methods $(0.04$ in OE and 0.07 in CL). While the MAPs from TG and RDG are not statistically different according to a Mann-Whitney $U$-test in the OE treatment $(z=1.156, p=0.248)$, they are significantly different in the CL treatment $(z=1.809, p=0.070){ }^{9}$

Overall, we observe that betrayal aversion is significant in our sample $(z=2.162, p=0.031)$, but less prominent compared to the original studies by Bohnet and co-authors (the average difference in MAPs between TG and RDG is 0.15 in Bohnet et al. 2008). However, we also notice that other studies using BZ design have found smaller size of betrayal aversion in line with our results $(0.07$ in Hong and Bohnet (2007), ${ }^{10} 0.08$ in Dreber et al. (2013), 0.07 Butler and Miller (2014), and 0.04 in Aimone et al. (2015) using a within-subject version of BZ design).

As a second step, we use regression analysis to directly compare the two methods. We report OLS regression estimates in Table 2. In Model (1), we regress the variable MAP on two treatment dummies: TG takes value 1 if the observation is collected in TG and zero otherwise and OE takes value 1 if the observation is collected using the OE version and zero otherwise. The estimated coefficient of TG suggests that MAPs increase significantly by $5 \%$ points in TG compared to RDG. This supports the presence of significant betrayal aversion in our entire sample. Additionally, the OE's coefficient reveals that MAPs elicited with the OE method are on average almost $6 \%$ points higher than the ones elicited with the CL method.

In Model (2) we control for the gender of the participants by including the dummy "Female". The inclusion of this control has no effect neither on the size nor on the significance of the treatment dummies and improves the overall fit of the model as suggested by the $R^{2}$. Interestingly, females report significantly higher MAPs than males both in TG and RDG. This is in line with the finding that women tend to be generally more averse to risks than men (Croson and Gneezy 2009).

Model (3) adds the interaction term between the variables TG and OE to Model (2). If the size of betrayal aversion elicited in CL is significantly higher than the one

\footnotetext{
9 Including in CL the subjects who switch more than once does not change the average difference between TG and RDG (0.07). However, betrayal aversion in CL reaches significance at $5 \%$ level $(z=2.041, p=0.041)$.

10 This statistic is not reported in the paper. We thank the authors for providing their data.
} 
Table 2 OLS regressions

\begin{tabular}{llll}
\hline & $(1)$ & $(2)$ & $(3)$ \\
\hline $\mathrm{TG}$ & $0.055^{* *}$ & $0.053 * *$ & $0.072 * *$ \\
& $(0.025)$ & $(0.024)$ & $(0.029)$ \\
$\mathrm{OE}$ & $0.059 * *$ & $0.060 * *$ & $0.079 * *$ \\
& $(0.025)$ & $(0.024)$ & $(0.033)$ \\
$\mathrm{TG} \times \mathrm{OE}$ & & & -0.039 \\
& & & $(0.049)$ \\
Female & & $0.108 * * *$ & $0.108 * * *$ \\
& & $(0.024)$ & $(0.024)$ \\
Intercept & $0.438 * * *$ & $0.383 * * *$ & $0.374 * * *$ \\
& $(0.019)$ & $(0.023)$ & $(0.025)$ \\
$R^{2}$ & 273 & 273 & 273 \\
\hline
\end{tabular}

elicited in OE, we should expect the interaction term to be negative and significant. Although the sign is indeed negative, the coefficient is not significant. This leads us to conclude that, although the two methods generate different distributions of values, this does not translate in statistically different estimates of the average treatment difference between TG and RDG.

We ask next which of the two methods is preferable to use in practice. Drawing from the results of Study 1, we notice that different conclusions on the significance of betrayal aversion could be reached depending on the method used. Hence, it is important to assess which of the two methods is better suited to elicit subjects' "true" valuations. We observe a general tendency of CL to generate less extreme values and less variation in the distribution of MAPs compared to OE. This could be due to a framing effect of the choice list, if subjects are led to switch more in the middle of the table. However, it could also be that subjects are less confused and these extreme values are in fact noisy valuations that the CL version helps to eliminate.

One way to assess whether this is the case is to check subjects' understanding in $\mathrm{OE}$ and CL. We conjecture that the comprehension of procedural aspects of the mechanism will be inversely related to the noise present in the elicited distributions of MAPs, and thus measuring subjects' understanding would reveal which method minimizes subjects' misconceptions.

\section{Study 2: assessing subjects' understanding of the instructions}

In Study 2, we conduct an online survey study where we compare the instructions of $\mathrm{OE}$ and CL. 


\subsection{Survey design and procedures}

Our participants were undergraduate students $(n=209)$ from the University of Nottingham who had not taken part in the experiments reported in Sect. 2. We recruited subjects through ORSEE (Greiner 2015). Upon their acceptance to participate in our online survey, subjects were told that the task was part of a research project and were invited to read an instruction document. After reading the instructions, they were asked a set of comprehension questions. Participants were informed before they started the task that they would not participate in the experiment. In Question 1, subjects had to answer the following: "How difficult do you think the instructions are?" on a scale from 0 to 10 , where 0 corresponds to "very difficult" and 10 to "very easy." Questions 2 and 3, each of which comprises three sub-items, were hypothetical scenarios where subjects in the role of the first movers were asked questions on the functioning of the BDM mechanism (see Online Appendix A.1 for the list of questions). Subjects were presented only the experimental instructions from TG. We assume that any difference would translate analogously to RDG. Participants were entered in a prize draw if they completed the task. There were two prizes of $£ 50$ each.

\subsection{Survey results}

In Table 3 we report the average response (standard deviation) to Question 1 and the percentage of mistakes in Question 2 and 3. The self-reported assessment of difficulty (Question 1) reveals that CL is perceived significantly easier than OE (MWU-test, $z=-4.307, p=0.000$ ). Furthermore, when we look at the comprehension questions, we also find that, for all sub-items of Question 2 and 3, the percentage of mistakes is significantly lower in CL than OE. These results suggest that CL is significantly easier for subjects and that we were successful in reducing subjects' confusion and mistakes.

Table 3 Answers to comprehension questions in CL and OE

\begin{tabular}{lllll}
\hline & $\mathrm{CL}(n=107)$ & $\mathrm{OE}(n=102)$ & Tests & $p$ value \\
\hline $\begin{array}{l}\text { Question } 1 \\
\text { Question } 2\end{array}$ & $6.40(2.34)$ & $4.90(2.40)$ & Mann-Whitney & 0.000 \\
(a) & $5.6 \%$ & $23.5 \%$ & $\chi^{2}$ test & 0.000 \\
(b) & $14 \%$ & $27.5 \%$ & $\chi^{2}$ test & 0.016 \\
(c) & $15 \%$ & $26.5 \%$ & $\chi^{2}$ test & 0.040 \\
Question 3 & & & & \\
(a) & $20.6 \%$ & $32.4 \%$ & $\chi^{2}$ test & 0.053 \\
(b) & $38.3 \%$ & $59.8 \%$ & $\chi^{2}$ test & 0.002 \\
(c) & $39.3 \%$ & $60.8 \%$ & $\chi^{2}$ test & 0.002 \\
\hline
\end{tabular}

All percentages are percentages of wrong answers 


\section{Conclusion}

In this paper, we have compared two implementations of the BDM mechanism for the elicitation of betrayal aversion in the BZ design. Overall, our results confirm the presumption that valuations elicited through the BDM mechanism may be biased due to subjects' mistakes and misconceptions of the incentive system. We further show that these mistakes can be reduced by employing a set of instructions that make the decision situation as clear as possible.

Despite no difference was found in the estimated size of betrayal aversion across $\mathrm{OE}$ and CL elicitations, our results indicate that valuations are significantly noisier in the OE version (Table 3) and different conclusions on the significance of betrayal aversion could be reached depending on the method used (Table 1). Hence, researchers should be careful in using a version of the mechanism that minimizes subjects' mistakes to make reliable inferences. In this sense, our CL version constitutes a significant improvement compared to $\mathrm{OE}$.

Acknowledgments I acknowledge support by CeDEx (Centre for Decision Research and Experimental Economics) at the University of Nottingham and from the grant European Research Council Advanced Investigator Grant Project COOPERATION to Simon Gächter (ERC-Adg 295707) which funded some of the experiments reported here. This paper is a revised version of Chapter 4 of my $\mathrm{PhD}$ thesis written under the supervision of Robin Cubitt and Simon Gächter at the University of Nottingham. I thank them for helpful discussions and encouragement. I also thank the editor Nikos Nikiforakis, two anonymous referees, Felix Kölle, Francesco Fallucchi, Chris Starmer, Martin Kocher, Daniele Nosenzo and Holger Gerhardt for helpful comments and suggestions.

\section{References}

Aimone, J. A., Ball, S. B., \& King-Casas, B. (2015). The betrayalaversion elicitation task: an individual level betrayal aversion measure. PLoS One, 10(9), e0137491. doi:10.1371/journal.pone.0137491.

Aimone, J. A., \& Houser, D. (2011). Beneficial betrayal aversion. PLoS One, 6(3), e17725. doi:10.1371/ journal.pone.0017725.

Aimone, J., \& Houser, D. (2012). What you don't know won't hurt you: a laboratory analysis of betrayal aversion. Exp Economics, 15(4), 571-588.

Aimone, J. A., \& Houser, D. (2013). Harnessing the benefits of betrayal aversion. J Econ Behav Organ, $89,1-8$.

Andersen, S., Harrison, G. W., Lau, M. I., \& Rutström, E. E. (2006). Elicitation using multiple price list formats. Exp Econ, 9(4), 383-405.

Andreoni, J. (1995). Cooperation in public-goods experiments-kindness or confusion? Am Econ Rev, 85(4), 891-904.

Bartling, B., Engl, F., Weber, RA (2015). Game form misconceptions are not necessary for a willingnessto-pay vs. willingness-to-accept gap. J Econ Sci Assoc, 1(1), 72-85.

Becker, G. M., Degroot, M. H., \& Marschak, J. (1964). Measuring utility by a single-response sequential method. Behav Sci, 9(3), 226-232.

Bohnet, I., Greig, F., Herrmann, B., \& Zeckhauser, R. (2008). Betrayal aversion. Evidence from Brazil, China, Oman, Switzerland, Turkey, and the United States. Am Econ Rev, 98(1), 294-310.

Bohnet, I., \& Zeckhauser, R. (2004). Trust, risk and betrayal. J Econ Behav Organ, 55(4), 467-484.

Butler, J.V., \& Miller, J.B. (2014). Social risk: the role of warmth and competence. Working Paper Series IGIER-Bocconi University n.522.

Cason, T. N., \& Plott, C. R. (2014). Misconceptions and game form recognition: challenges to theories of revealed preference and framing. J Political Econ, 122(6), 1235-1270. 
Croson, R., \& Gneezy, U. (2009). Gender differences in preferences. J Econ Lit, 47(2), 1-27.

Cubitt R, Gächter S, Quercia S (2015) Conditional cooperation and betrayal aversion. CeDEx discussion paper, 2015-14.

Dreber, A., Rand, D.G., Wernerfelt, N., Worrell, P.R., Zeckhauser, R.J. (2013). The decisions of entrepreneurs and their agents: revealed levels of risk Aversion and betrayal aversion. Harvard Kennedy School Research Working Paper Series-RWP13-016.

Gigerenzer, G., \& Hoffrage, U. (1995). How to improve bayesian reasoning without instructionfrequency formats. Psychol Rev, 102(4), 684-704.

Greiner, B. (2015). Subject pool recruitment procedures: organizing experiments with ORSEE. J Econ Sci Assoc, 1(1), 114-125.

Hao, L., \& Houser, D. (2012). Belief elicitation in the presence of naïve respondents: an experimental study. J Risk Uncertainty, 44(2), 161-180.

Harrison, G. W., \& Rutstrom, E. E. (2008). Risk aversion in the laboratory. Res Exp Econ, 12, 41-196.

Hong, K., \& Bohnet, I. (2007). Status and distrust: the relevance of inequality and betrayal aversion. $J$ Econ Psychol, 28(2), 197-213.

Houser, D., \& Kurzban, R. (2002). Revisiting kindness and confusion in public goods experiments. Am Econ Rev, 92(4), 1062-1069.

Selten, R. (1967). Die Strategiemethode zur Erforschung des eingeschränkt rationalen Verhaltens im Rahmen eines Oligopolexperimentes. In H. Sauermann (Ed.), Beiträge zur experimentellen Wirtschaftsforschung (pp. 136-168). Mohr (Paul Siebeck): J.C.B. 